\title{
Electrochemical Control of Bioluminescence by Blocking the Adsorption of the Bacterial Luciferase Using a Mercaptobipyridine Self-assembled Monolayer
}

\author{
Shinya YamasakI, ${ }^{* 1 \dagger}$ Shuto YAMAda, ${ }^{* 2}$ Hiroyuki TAKemura, ${ }^{* 3}$ and Kô TAKeHARA $* 4$ \\ *1 Faculty of Pure and Applied Sciences and Center for Research in Isotopes and Environmental Dynamics, \\ University of Tsukuba, 1-1-1 Tennodai, Tsukuba, Ibaraki 305-8577, Japan \\ *2 Department of Applied Chemistry, School of Applied Sciences National Defense Academy, 1-10-20 Hashirimizu, \\ Yokosuka 239-8686, Japan \\ *3 Department of Chemical and Biological Science, Faculty of Science, Japan Women's University, 2-8-1 Mejirodai, \\ Bunkyo, Tokyo 112-8681, Japan \\ *4 Department of Chemistry, Kyushu University, 744 Motooka, Nishi, Fukuoka 819-0395 Japan
}

\begin{abstract}
An $N$-butyl- $N^{\prime}$-(4-mercaptobutyl)-4,4'-bipyridinium (4BMBP) was modified on a gold electrode to improve the electrochemical control of the bacterial luciferase (BL) luminescence system. The 4BMBP-modified gold electrode (4BMBP/Au) was able to prevent the adsorption of $\mathrm{BL}$ on the electrode surface, and enhanced the electrochemical regeneration rate of the reduced flavin mononucleotide $\left(\mathrm{FMNH}_{2}\right)$, which is one of the substrates of the BL luminescence reaction. By using the 4BMBP/Au, the luminescence intensity increased by about $27 \%$ compared to that of a bare gold electrode (bare $\mathrm{Au}$ ). Moreover, the modified electrode improved the time required for analysis because the modified layer prevented BL adsorption. Even without a refreshing procedure for each measurement, a constant luminescence intensity could be observed, and the analysis time was reduced to half (about $10 \mathrm{~min}$ ) for one sample. The 4BMBP/Au is not only useful to control of the BL luminescence system, but also for electrochemical measurements in the presence of proteins.
\end{abstract}

Keywords Electrochemically-controlled luminescence, flavin mononucleotide, cyclic voltammetry, protein adsorption

(Received July 21, 2016; Accepted October 13, 2016; Published March 10, 2017)

\section{Introduction}

Luminescence enzymes extracted from the firefly and bacteria have been used as a model of a membrane protein to elucidate the inhibitory mechanism of general anesthesia, which is in the category of hydrophobic molecules. ${ }^{1-6}$ The effect of hydrophobic molecules, such as terminally-substituted normal alkyl compounds, on the bacterial luciferase (BL) bioluminescence has been reported in our previous studies. ${ }^{5,6}$ The BL reaction requires a reduced flavin mononucleotide $\left(\mathrm{FMNH}_{2}\right)$ as one of the substrates. ${ }^{7}$ However, the $\mathrm{FMNH}_{2}$ is rapidly oxidized to oxidized flavin (FMN) by dissolved oxygen in an aqueous solution. To overcome this problem, we have established the electrochemical regeneration system of FMN to generate the BL luminescence shown by the following equations: ${ }^{8,9}$

$$
\begin{array}{r}
\mathrm{FMN}+2 \mathrm{H}^{+}+2 \mathrm{e}^{-} \longrightarrow \mathrm{FMNH}_{2} \text { (electrode reaction) } \\
\mathrm{FMNH}_{2}+\mathrm{O}_{2}+\mathrm{C}_{11} \mathrm{H}_{23} \mathrm{CHO} \longrightarrow \\
\mathrm{FMN}+\mathrm{H}_{2} \mathrm{O}+\mathrm{C}_{11} \mathrm{H}_{23} \mathrm{COOH}+h v
\end{array}
$$

When a metal electrode, such as bare Au, was used as the

$\dagger$ To whom correspondence should be addressed.

E-mail: s-yamasaki@ied.tsukuba.ac.jp working electrode, the $\mathrm{BL}$ reaction was inhibited by the adsorption of BL molecules on the electrode surface. The adsorbed BL blocked the FMN reduction based on Eq. (1). It produces a poor accuracy and time consuming analysis of the inhibitory potency of the hydrophobic molecules on the BL luminescence reaction. Therefore, cleaning the electrode surface has been required for each measurement. To improve the BL system, we focused on a modification of the electrode surface. The self-assembled monolayer (SAM) modified electrode is a well-examined technique for various electrochemical systems. By introducing the functional thiol compound on the electrode surface, various improvements can be achieved. ${ }^{10}$

In the present study, we used the $N$-butyl- $N^{\prime}$-(4-mercaptobutyl)$4,4^{\prime}$-bipyridinium ion (4BMBP), shown in Fig. 1, as a component of SAM, and applied it for electrochemical control of the BL luminescence system. It can be expected that the bipyridinium

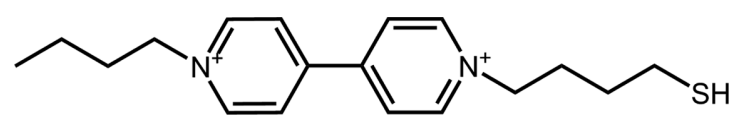

Fig. 1 Structure of $N$-butyl- $N^{\prime}$-(4-mercaptobutyl)-4,4'-bipyridinium ion (4BMBP). The counter anions were dihexafluorophosphate in the present study. 
unit functions as a mediator for the reduction process of the FMN. The standard redox potential of the bipyridinium compound is about $-0.31 \mathrm{~V}^{11}$ (vs. $\mathrm{Ag} / \mathrm{AgCl}$ ), and that of $\mathrm{FMN}$ is about $-0.43 \mathrm{~V} .{ }^{12}$ Although the redox potential of 4BMBP is higher than that of FMN, electron transfer from 4BMBP to FMN is expected to be possible at a potential lower than the FMN reduction:

$$
\begin{aligned}
& \text { bpy } \left.(\mathrm{Ox})+2 \mathrm{e}^{-} \rightleftharpoons \text { bpy(red }\right) \\
& \text { bpy }(\text { red })+\mathrm{FMN}+2 \mathrm{H}^{+} \rightleftharpoons \mathrm{bpy}(\mathrm{Ox})+\mathrm{FMNH}_{2}
\end{aligned}
$$

where bpy(ox) and bpy(red) indicate the redox state of 4BMBP modified on the electrode surface. The SAM modified on the electrode is also expected to prevent the adsorption of BL on the electrode surface. By using the 4BMBP SAM modified gold electrode (4BMBP/Au), we examined (1) the redox behavior of the bipyridinium unit in 4BMBP/Au, (2) the effect of SAM on the redox behavior of FMN, (3) the blocking of the BL adsorption on the electrode surface by SAM, and (4) the usefulness of 4BMBP/Au for the BL luminescence system.

\section{Experimental}

\section{SAM preparation}

For the voltammetric measurement, the SAM was modified on a gold disk $(\phi=1.6 \mathrm{~mm})$ electrode $(\mathrm{SAM} / \mathrm{Au})$ by immersing it in $1 \mathrm{mmol} \mathrm{dm}^{-3}$ thiol in $10 \mathrm{~cm}^{3}$ of acetonitrile for 3 days at room temperature $\left(25 \pm 1^{\circ} \mathrm{C}\right)$. For the bioluminescence measurement, the SAM was modified on a gold mesh (100 mesh) electrode $(\mathrm{SAM} / \mathrm{Au})$. The effective electrode area was determined to be $4.72 \mathrm{~cm}^{3}$ by using $\mathrm{K}_{4} \mathrm{Fe}(\mathrm{CN})_{6}$. After washing the gold mesh electrode in a piranha solution $\left(30 \% \mathrm{H}_{2} \mathrm{O}_{2}: \mathrm{H}_{2} \mathrm{SO}_{4}\right.$ $\left.=1: 3(\mathrm{v} / \mathrm{v}), 60^{\circ} \mathrm{C}\right)$ for $1 \mathrm{~h}$, the electrode was immersed in the 4BMBP solution for 3 days at room temperature. To examine the effect of 4BMBP on the BL luminescence intensity, hexane thiol (HXT) was mixed in the 4BMBP solution for a control experiment. Three kinds of thiol solutions were prepared, as follows: 4BMBP:HXT = 1:0 (4BMBP/Au), 1:1 (4BMBP-HXT/ $\mathrm{Au})$ and $0: 1$ in molar ratio $(\mathrm{HXT} / \mathrm{Au})$.

\section{Cyclic voltammetry of SAM modified electrode}

The electrode reaction of 4BMBP/Au was evaluated by cyclic voltammetry. The SAM/Au was used as the working electrode. The scan rate was $10 \mathrm{mV} \mathrm{s}^{-1}$. $\mathrm{Pt}$ wire and a $\mathrm{Ag} / \mathrm{AgCl}$ (in $3 \mathrm{~mol} \mathrm{dm}^{-3} \mathrm{NaCl}$ solution) electrode were used as the counter and reference electrodes, respectively. A $50 \mathrm{mmol} \mathrm{dm}^{-3}$ phosphate buffer solution adjusted at $\mathrm{pH} 7.0$ containing $100 \mathrm{mmol} \mathrm{dm}^{-3} \mathrm{KCl}$ as the supporting electrolyte was used in this measurement. For all of the experiments in this study, the $\mathrm{pH}$ value was kept constant at 7.0, because the optimal $\mathrm{pH}$ of BL is between 8.5 and 6.5, and many physicochemical examinations using $\mathrm{BL}$ have been done at $\mathrm{pH} 7.0 .^{13}$

\section{Effect of SAM on FMN redox behavior and BL adsorption}

Cyclic voltammetry was performed using a solution of $1.0 \mathrm{mmol} \mathrm{dm}^{-3} \mathrm{FMN}$ containing $0.1 \mathrm{mg} \mathrm{cm}^{-3} \mathrm{BL}, 100 \mathrm{mmol} \mathrm{dm}^{-3}$ $\mathrm{KCl}$ and $50 \mathrm{mmol} \mathrm{dm}^{-3}$ phosphate buffer ( $\mathrm{pH}$ 7.0). Either the $\mathrm{SAM} / \mathrm{Au}$ or the bare $\mathrm{Au}$ electrode was used as the working electrode. Pt wire and $\mathrm{Ag} / \mathrm{AgCl}$ were used as the counter and reference electrodes, respectively. The measurement was started 5 min after immersing the working electrode into the solution in order to examine the effect of BL adsorption on the electrode surface. The scan rate was $10 \mathrm{mV} \mathrm{s}^{-1}$.

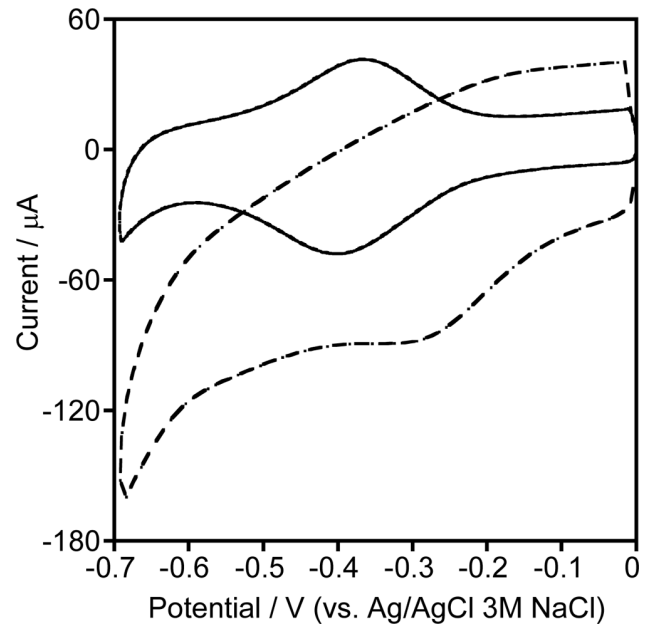

Fig. 2 Cyclic voltammogram of 4BMBP/Au-mesh in $50 \mathrm{mmol} \mathrm{dm}^{-3}$ phosphate buffer and $100 \mathrm{mmol} \mathrm{dm}^{-3} \mathrm{KCl}$ solution. The solid line and dashed line indicate the result of the $4 \mathrm{BMBP} / \mathrm{Au}$ and the bare $\mathrm{Au}$, respectively. The $\mathrm{pH}$ value was adjusted to 7.0. The scan rate was $100 \mathrm{mV} \mathrm{s}^{-1}$. The Au mesh electrode was used in this experiment.

Application to BL luminescence system

The BL luminescence intensity was measured by a previously reported system. ${ }^{6}$ BL extracted from Vibrio fischeri [EC: 1.14.14.3] (Sigma-Aldrich Co. LLC., USA) was used in this study. The 4BMBP/Au was used as a working electrode. The luminescence measurement was performed using a homemade flow electrochemical luminescence cell. FMN was reduced by linear sweep voltammetry and the corresponding BL luminescence was observed by a photomultiplier. The potential was scanned from -0.30 to $-0.65 \mathrm{~V}(v s . \mathrm{Ag} / \mathrm{AgCl})$ at a scan rate of $10 \mathrm{mV} \mathrm{s}^{-1}$.

\section{Effect of a SAM modified electrode on repeated BL luminescence measurements}

The 4BMBP/Au was applied to the BL luminescence system. In order to examine the effectiveness of the electrode in the repetition experiments, the variation in the maximum luminescence intensity was measured during repeated measurements. The 4BMBP/Au and bare Au were used as the working electrode, and the BL luminescence was recorded without refreshing the electrode. The measurement was repeated 4 times for each electrode. The BL luminescence reaction was controlled by constant potential electrolysis at $-0.70 \mathrm{~V}$ (vs. $\mathrm{Ag} / \mathrm{AgCl})$.

\section{Results and Discussion}

\section{Electrochemical behavior of SAM modified electrode}

Figure 2 shows a cyclic voltammogram of the $4 \mathrm{BMBP} / \mathrm{Au}$ in a $50 \mathrm{mmol} \mathrm{dm}^{-3}$ phosphate buffer solution. The dashed line indicates the result of the bare mesh electrode, and the solid line indicates that of the 4BMBP/Au. The cathodic peak around $-0.3 \mathrm{~V}$ for the bare electrode resulted from the reduction of dissolved oxygen. On the other hand, the redox peaks corresponding to the redox reaction of the bipyridinium unit on the electrode surface were observed at around $-0.34 \mathrm{~V}$. This result clearly indicated that the redox peaks were caused by the reaction of the electroactive species adsorbed on the electrode surface. The redox behaviors of similar bipyridinium SAM 

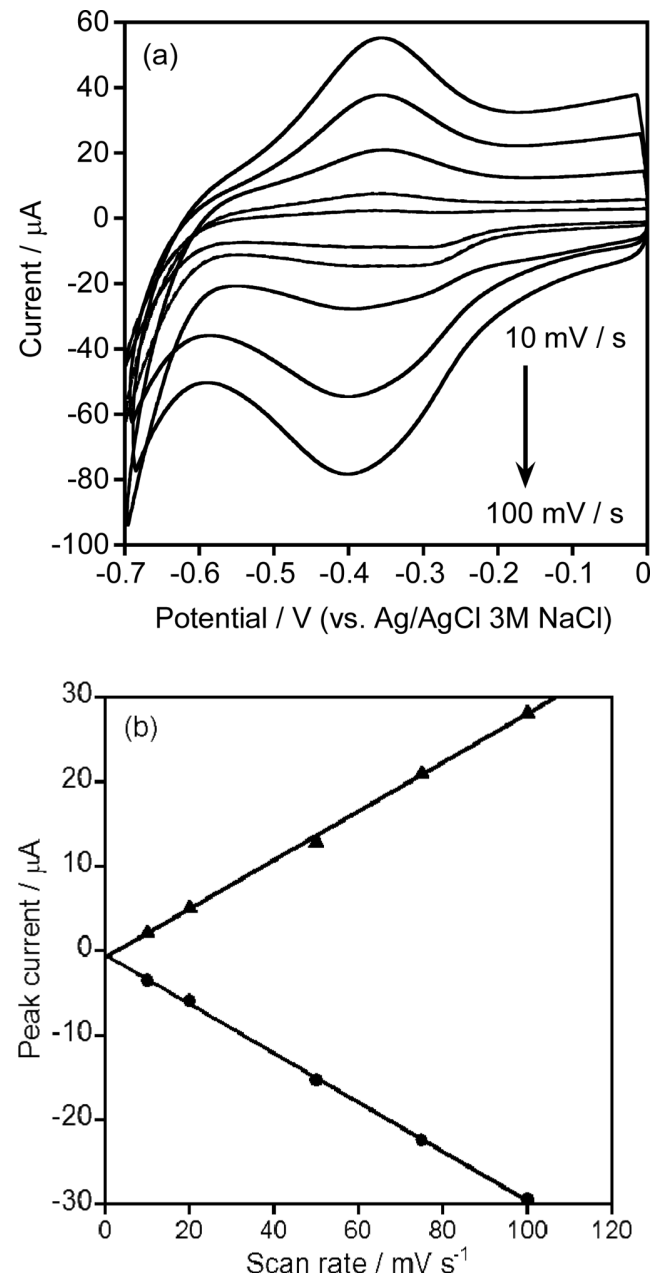

Fig. 3 (a) Cyclic voltammograms of the 4BMBP/Au mesh electrode in $50 \mathrm{mmol} \mathrm{dm}^{-3}$ phosphate buffer and $100 \mathrm{mmol} \mathrm{dm}^{-3} \mathrm{KCl}$ solution at $\mathrm{pH}$ 7.0. The scan rates were 10, 20, 50, 75 and $100 \mathrm{mV} \mathrm{s}^{-1}$. (b) Scan rate dependence on the redox currents. The closed circles indicate the cathodic peak currents and the closed triangles indicate the anodic peak currents. The redox currents were obtained from Fig. 3(a). The solid lines indicate a first-order regression line.

modified electrodes have been reported. ${ }^{11,14}$ Although the bipyridinium thiol having a different alkyl chain length of the terminal group was used in the previous study, the redox peaks obtained in the present study were similar to those of the previous one ${ }^{11}(-0.31 \mathrm{~V}$, corrected for $\mathrm{Ag} / \mathrm{AgCl}$ electrode). In the literature, ${ }^{11}$ the redox peaks correspond to the one-electron reduction of a bipyridinium unit shown in Eq. (5), where bpy ${ }^{2+}$ and bpy $^{\circ+}$ denote the divalent bipyridinium cation and the monovalent bipyridinium radical, respectively.

$$
\mathrm{bpy}^{2+}+\mathrm{e}^{-} \rightleftharpoons \mathrm{bpy}^{++}
$$

The values of the anodic and cathodic currents were almost the same as shown in Fig. 2. Therefore, the bpy*+ radical formed by the cathodic reaction was not spontaneously reoxidized to the $\mathrm{bpy}^{2+}$. Figure 3(a) shows cyclic voltammograms of 4BMBP/Au in $50 \mathrm{mmol} \mathrm{dm}{ }^{-3}$ phosphate buffer at scan rates of $10,20,50,75$ and $100 \mathrm{mV} \mathrm{s}^{-1}$, respectively. Both the cathodic peak currents and the anodic peak currents increased with the scan rate. The peak currents were plotted versus the scan rate, as shown in Fig. 3(b). Linear lines having an intercept of zero are shown in

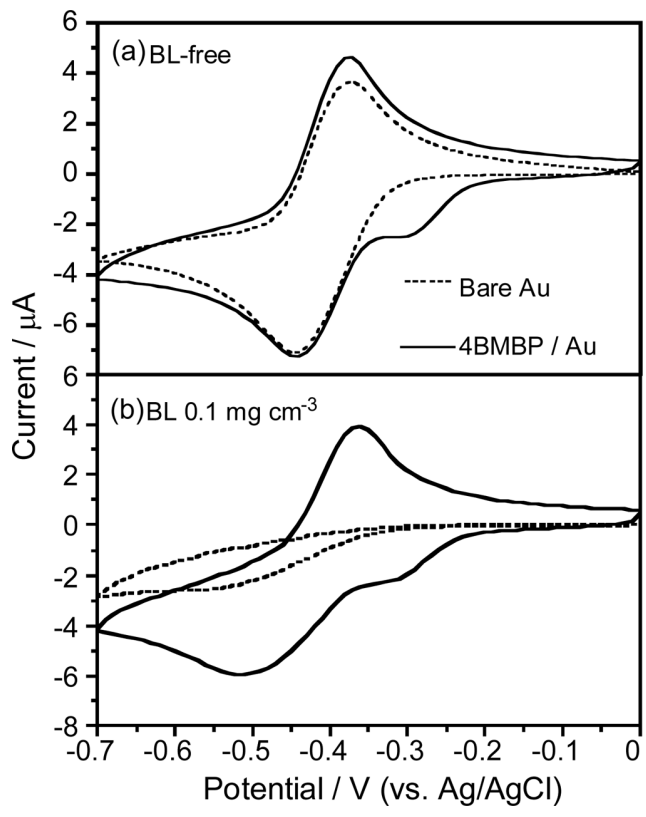

Fig. 4 Cyclic voltammograms of 4BMBP/Au (solid line) and bare $\mathrm{Au}$ (dotted line) in $1.0 \mathrm{mmol} \mathrm{dm}^{-3} \mathrm{FMN}$ solution containing no BL (a), and $0.1 \mathrm{mg} \mathrm{cm}^{-3} \mathrm{BL}$ (b). A $50 \mathrm{mmol} \mathrm{dm}^{-3}$ phosphate buffer and $100 \mathrm{mmol} \mathrm{dm}^{-3} \mathrm{KCl}$ were added to the sample solution as a $\mathrm{pH}$ buffer and supporting electrolyte, respectively. The scan rate was $10 \mathrm{mV} \mathrm{s}^{-1}$.

Fig. 3(b); the potential difference between the anodic peak and cathodic peak was less than $10 \mathrm{mV}$, indicating the redox reaction of the adsorbed SAM rather than the diffusion of the redox active species.

Effect of a SAM modified electrode on the FMN redox reaction and $B L$ luminescence reaction

After the 4BMBP was successfully immobilized on the electrode surface, the redox behavior of FMN was then measured using 4BMBP/Au. Figure 4(a) shows cyclic voltammograms of the $1.0 \mathrm{mmol} \mathrm{dm}^{-3} \mathrm{FMN}$ solution obtained using bare $\mathrm{Au}$ and 4BMBP/Au. In the case of the bare Au electrode (dotted line), only one cathodic peak corresponding to the reduction of FMN was observed. On the contrary, two cathodic peaks $(-0.30$ and $-0.45 \mathrm{~V})$ and one anodic peak $(-0.39 \mathrm{~V})$ were observed for the 4BMBP/Au. The cathodic peak at around $-0.30 \mathrm{~V}$ can be ascribed to a reduction of the bipyridinium unit, as shown in Fig. 2. These results indicated that a small molecule, such as FMN, is able to penetrate into the 4BMBP SAM because of the loosely packed SAM layer. The redox behavior of the small compound has also been confirmed using the $\mathrm{Fe}(\mathrm{CN})_{6}{ }^{4-}$ ion, and clear redox currents could be observed in addition to those of the thiol SAM (data are shown in Fig. S1, Supporting Information).

Figure 4(b) shows cyclic voltammograms of the $1 \mathrm{mmol} \mathrm{dm}^{-3}$ FMN solution containing $0.1 \mathrm{mg} \mathrm{cm}^{-3} \mathrm{BL}$ using a bare $\mathrm{Au}$ (dashed line) and 4BMBP/Au (solid line). In the presence of $\mathrm{BL}$, the cathodic peak at $-0.52 \mathrm{~V}$ was small for bare $\mathrm{Au}$ as compared to the 4BMBP/Au. These voltammograms were recorded after a 5-min immersion of the electrodes in the $\mathrm{BL}$ solution. Therefore, the BL was adsorbed onto the bare $\mathrm{Au}$ surface, which prevented the redox reaction of FMN. At the 4BMBP/Au, in contrast, a well-defined current response was observed. Although the modified SAM is considered to reduce the $\mathrm{BL}$ adsorption, the FMN redox reaction was not reversible 


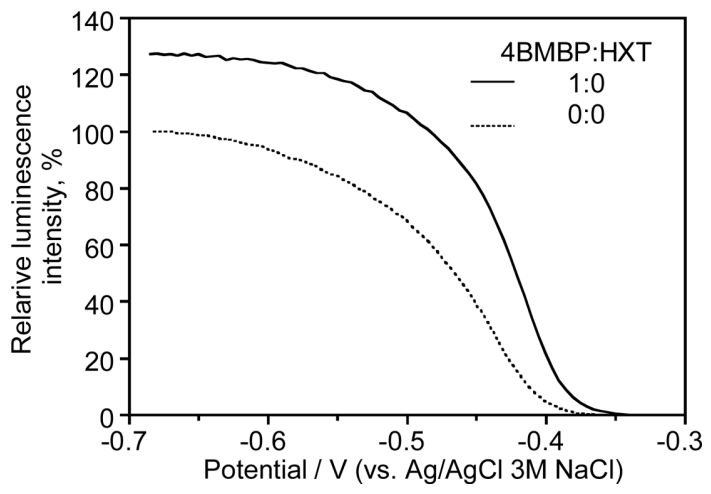

Fig. 5 Effect of the electrolysis potential on the relative luminescence intensity. Mixing ratios of 4BMBP and HXT were 1:0 (4BMBP/Au, solid line), 0:0 (bare Au, dotted line). The results of 1:1 (4BMBP$\mathrm{HXT} / \mathrm{Au}$ ) and 0:1 (HXT/Au) are not shown (no luminescence).

(anodic peak: $-0.38 \mathrm{~V}$ and cathodic peak: $-0.52 \mathrm{~V}$ ). This result indicated that although the SAM prevented the adsorption of $\mathrm{BL}$, it also affected the FMN transport near the electrode surface. Although the reaction rate of FMN at 4BMBP/Au decreased by the presence of BL, it still proceeded. This will be a great advantage for the application of the $4 \mathrm{BMBP} / \mathrm{Au}$ to the $\mathrm{BL}$ luminescence system to maintain the regeneration of $\mathrm{FMNH}_{2}$ and, in addition, prevent contamination of the electrode surface.

\section{Application of 4BMBP modified electrode for the BL luminescence reaction}

The 4BMBP/Au was applied to the BL luminescence system. Figure 5 shows the effect of the electrode potential on the $\mathrm{BL}$ luminescence intensity. A potential sweep was performed by linear sweep voltammetry at a $10 \mathrm{mV} \mathrm{s}^{-1}$ scan rate. The luminescence intensity was normalized by the luminescence intensity at $-0.65 \mathrm{~V}$ using the bare $\mathrm{Au}$. In the case of the bare $\mathrm{Au}$, although the reduction peak of FMN was not observed, as shown in Fig. 4(b), the BL luminescence was observed at a negative potential lower than $-0.38 \mathrm{~V}$ (Fig. 5, dashed line). The luminescence intensity at the bare $\mathrm{Au}$ reached a constant value at around $-0.65 \mathrm{~V}$, in good agreement with the reduction potential of FMN. In the case of 4BMBP/Au, a similar potential dependence of the luminescence intensity was also observed. However, the maximum luminescence intensity was $27 \%$ higher at the 4BMBP/Au than at the bare Au. This result indicated the effective reduction of FMN at the modified electrode, as shown in Fig. 4(b). Although this enhancement of the luminescence intensity seems to disagree with the FMN reduction rate observed in the cyclic voltammograms shown in Fig. 4(b), the 4BMBP/Au is useful to effectively promote the BL luminescence reaction. To clarify the effect of 4BMBP/Au, the 4BMBP SAM was replaced with the hexane thiol (HXT) SAM and 4BMBPHXT mixed SAM; however, the BL luminescence was not observed at any of the HTX/Au and 4BMBP-HXT/Au electrodes. This is probably caused by the formation of a densely packed HXT SAM in the gap of 4BMBP SAM, even in the case of 4BMBP:HXT = 1:1. In the other case, the HXT may preferentially adsorb on the electrode compared to 4BMBP under the conditions of the present thiol concentration.

Finally, to examine the practicality of the $4 \mathrm{BMBP} / \mathrm{Au}$, repeated measurements were performed. Figure 6 shows the effect of rinsing the electrode surface for each measurement. The open circle and the closed square indicate the results of a bare $\mathrm{Au}$ and the 4BMBP/Au, respectively. The luminescence

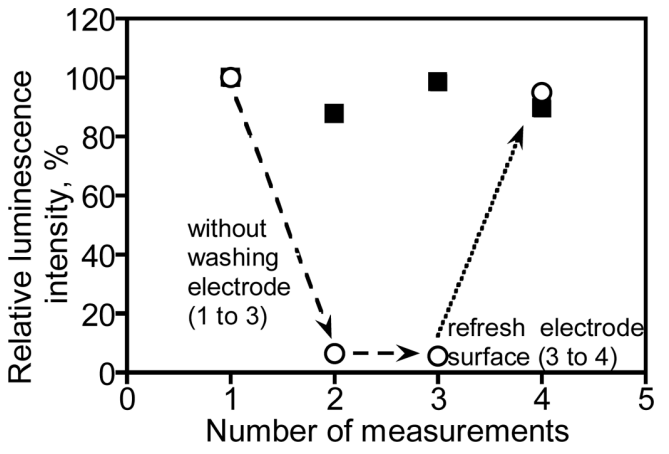

Fig. 6 Effect of electrode cleaning on the reproducibility of the luminesence intensity for 4BMBP/Au (closed square) and bare $\mathrm{Au}$ (open circle). For the $4 \mathrm{BMBP} / \mathrm{Au}$, the reproducible luminescence intensities were observed without washing the electrode surface. The variation in the luminescence intensity was about $5 \%$ for 4 measurements, which is in the acceptable range. In the case of the bare $\mathrm{Au}$, the electrode surface was washed with ethanol before the first and fourth measurements. If the bare $\mathrm{Au}$ was not washed during each measurement, the luminescence intensity significantly decreased as shown in the second and third measurements. The washing of the bare $\mathrm{Au}$ surface regenerates the BL reaction, as shown in the fourth measurement.

intensity was normalized by the luminescence intensity of the first measurement. In the case of the bare $\mathrm{Au}$, the luminescence intensity significantly decreased with repeated measurements without washing the electrode surface (from first to third measurements). After the third measurement, the bare Au was rinsed for $5 \mathrm{~min}$ with sonication in ethanol. This procedure resulted in regenerating the luminescence intensity during the fourth measurement. In the case of the $4 \mathrm{BMBP} / \mathrm{Au}$, almost the same luminescence intensity was observed during repeated measurements without washing the electrode surface. The relative standard deviation of 4 repeated measurements was $5.4 \%$, which is not significantly different from those of the bare $\mathrm{Au}$ with electrode cleaning for each measurement. Therefore, the $4 \mathrm{BMBP} / \mathrm{Au}$ is applicable to the $\mathrm{BL}$ luminescence system and is strongly expected to reduce the required time for analysis by half (about $10 \mathrm{~min}$ ) compared to the previous method. 5,6

\section{Conclusions}

The 4BMBP-modified Au electrode (4BMBP/Au) was prepared to prevent the adsorption of $\mathrm{BL}$ on the electrode surface and to promote FMN reduction. The 4BMBP/Au showed well-defined redox reactions of the bipyridinium unit and FMN, even in the presence of $\mathrm{BL}$. The 4BMBP/Au increases the reduction rate of FMN to form $\mathrm{FMNH}_{2}$. The BL luminescence intensity increased about $27 \%$ at the $4 \mathrm{BMBP} / \mathrm{Au}$ as compared to that of the bare $\mathrm{Au}$. These results can be acsribed to the effective reduction of FMN. In addition, the 4BMBP/Au showed an effective FMN reduction by protecting the $\mathrm{BL}$ adsorption on the electrode surface. It can be concluded that $4 \mathrm{BMBP} / \mathrm{Au}$ is a useful electrode to control the BL luminescence reaction. Moreover, the redox-active bipyridinium unit of $4 \mathrm{BMBP} / \mathrm{Au}$ will be applicable for other electrochemical analyses of samples containing proteins. 


\section{Supporting Information}

This material is available free of charge on the Web at http:// www.jsac.or.jp/analsci/.

\section{References}

1. I. Ueda and H. Kamaya, Anesthesiology, 1973, 38, 425.

2. N. P. Franks and W. R. Lieb, Nature, 1984, 310, 599.

3. N. P. Franks, A. Jenkins, E. Conti, W. R. Lieb, and P. Brick, Biophys. J., 1998, 75, 2205.

4. I. Ueda and A. Suzuki, Biochim. Biophys. Acta, 1998, 1380, 313.

5. S. Yamasaki, S. Yamada, and K. Takehara, Anal. Sci., 2013 , 29, 41.
6. S. Yamasaki, S. Yamada, and K. Takehara, Anal. Sci., 2011, 27, 357.

7. J. W. Hastings and Q. H. Gibson, J. Biolog. Chem., 1963, 238, 2537.

8. S. Yamasaki, S. Nakashima, S. Yamada, and K. Takehara, Bioelectrochemistry, 2009, 75, 67.

9. K. Takehara, S. Nakashima, S. Yamasaki, and S. Matsuoka, Chem. Lett., 2007, 36, 1358.

10. D. Chen and J. Li, Surf. Sci. Reports, 2006, 61, 445.

11. J. Li, G. Cheng, and S. Dong, Thin Solid Films, 1997, 293, 200.

12. S. L. J. Tan, J. M. Kan, and R. D. Webster, J. Phys. Chem. $B, 2013,117,13755$.

13. L. J. Blum and P. R. Coulet, Anal. Chim. Acta, 1984, 161 355.

14. B. Han, Z. Li, T. Wandlowski, A. Blaszczyk, and M. Mayor, J. Phys. Chem. C, 2007, 111, 13855. 